\title{
SPIN-LATTICE RELAXATION IN CERIUM MAGNESIUM NITRATE NO PARTIAL LATTICE PARTICIPATION OBSERVED
}

\author{
J. A. OVERWEG, J. FLOKSTRA and G. J. GERRITSMA \\ Twente University of Technology, P.O. Box 217, Enschede, The Netherlands
}

\begin{abstract}
Dynamic susceptibility measurements on thermally isolated cerium magnesium nitrate show that all lattice oscillators participate in the Orbach spin-lattice relaxation process. The observed environmental influence on the relaxation behaviour when the crystal is in direct contact with liquid helium can be explained with a thermal conduction model.
\end{abstract}

\section{Introduction}

In the direct and Orbach spin-lattice relaxation processes only narrow phonon bands are involved in the recovery. Phonon-bottleneck theories assume that the surplus energy in the resonant bands is transferred either to the other phonon modes or directly to the bath. Which one of these two relaxation paths is actually effective can be deduced from dynamic susceptibility measurements by comparing the results when the crystal is placed in liquid helium with those when it is thermally isolated (vacuum). In the latter case the difference between the isothermal and adiabatic susceptibility, $\chi_{\mathrm{T}}-$ $\chi_{\mathrm{ad}}$, is a factor $\gamma$ smaller [1]:

$$
\begin{aligned}
\gamma & =\left(\chi_{\mathrm{T}}-\chi_{\mathrm{ad}}\right)_{\mathrm{vac}} /\left(\chi_{\mathrm{T}}-\chi_{\mathrm{ad}}\right)_{\mathrm{liq}} \\
& =C_{\mathrm{L}}^{*} /\left(C_{\mathrm{H}}+C_{\mathrm{L}}^{*}\right) .
\end{aligned}
$$

$C_{\mathrm{H}}$ is the spin specific heat at constant field and $C_{\mathrm{L}}^{*}$ is the effective lattice specific heat. If the entire lattice is coupled to the spin system one would find a $C_{\mathrm{L}}^{*}$ value equal to the calorimetric value $C_{\mathrm{L}}$ whereas if only narrow phonon bands are coupled to the spins the measured $C_{\mathrm{L}}^{*}$ would be in the order of $10^{-3} C_{\mathrm{L}}$. By performing these "liquid-vacuum" measurements on potassium chromium alum, where the direct process is dominant, we found the lattice participation ratio $C_{\mathrm{L}}^{*} / C_{\mathrm{L}}$ to be neither 1 nor in the order of $10^{-3}$ but varying from 0.5 at $2 \mathrm{~K}$ to 0.9 at $4.2 \mathrm{~K}$ [1]. In order to investigate whether a similar partial lattice participation also occurs in Orbach systems we examined the relaxation behaviour of cerium magnesium nitrate (CMN), where the resonant phonon bands lie on the high frequency side of the phonon spectrum.

\section{Experimental results}

Using a mutual inductance bridge we carried out a series of liquid-vacuum experiments on a
CMN single crystal (size $8 \times 3 \times 2 \mathrm{~mm}^{3}$ ) at temperatures between 2 and $4.2 \mathrm{~K}$. The magnetic field (up to $20 \mathrm{kOe}$ ) was applied perpendicular to the $c$-axis of the crystal. Although the spin-lattice relaxation time constant $\tau_{\mathrm{sl}}$ which obeys $\tau_{\mathrm{sl}}=A H^{\frac{1}{2}}$ $\exp (36.25 / T)[2]$ is very small at $T=4.2 \mathrm{~K}(\tau=6$ $\times 10^{-6} \mathrm{~s}$ at $\left.H=20 \mathrm{kOe}\right)$ we still observed substantial relaxation effects in our liquid experiments at frequencies as low as $30 \mathrm{~Hz}$ and we could not reach the isothermal limit of the dispersion (fig. 1). These low-frequency relaxation effects, which become particularly important at fields above $10 \mathrm{kOe}$, are not due to the intrinsic relaxation process but originate from a limited heat transfer in the lattice or bath. Our thermal conduction model [1] predicts that the thermal conductivity $\lambda_{\mathrm{He}}$ of liquid helium leads to a bottleneck in the energy transfer when $r_{0}^{2} C_{\mathrm{H}} / \lambda_{\mathrm{He}} \tau_{\mathrm{sl}}>1$, where $r_{0}$ is the effective radius of the sample (the lattice thermal conductivity $\lambda_{\mathrm{L}}$ of CMN is much larger than $\lambda_{\mathrm{He}}$ ). At $T=4.2 \mathrm{~K}$ and $H=17.9 \mathrm{kOe}$ this ratio equals $1.6 \times 10^{4}$ so the model predicts a large influence of the thermal conductivity of the helium on the observed susceptibility. With the thermal conduction model we calculated the dispersion and absorption curves at this temperature and field. In the calculations the crystal was represented by a cylinder with a radius of $1.5 \mathrm{~mm}$, a specific heat $C_{\mathrm{L}}=1.26 \times 10^{3} \mathrm{~J} / \mathrm{m}^{3} \mathrm{~K}$ [3] and a thermal conductivity $\lambda_{L}=1.50 \times 10^{3}$ $\mathrm{W} / \mathrm{mK}$ [4]. The spin specific heat $C_{H}$ and the paramagnetic saturation factor $K=\chi_{\mathrm{T}}(H) / \chi_{\mathrm{T}}(0)$ were calculated with the molecular field model as $C_{\mathrm{H}}=1.45 \times 10^{3} \mathrm{~J} / \mathrm{m}^{3} \mathrm{~K}$ and $K=0.94$. For the spin-lattice relaxation time we used the observed value $7.5 \times 10^{-6} \mathrm{~s}$. Finally, the bath was representedby $C_{\mathrm{He}}=5.65 \times 10^{5} \mathrm{~J} / \mathrm{m}^{3} \mathrm{~K}$ and $\lambda_{\mathrm{He}}=2.66$ $\times 10^{-2} \mathrm{~W} / \mathrm{mK}$ and the thermal resistance between the crystal and bath neglected (all these data are at $T=4.2 \mathrm{~K}$ and $H=17.9 \mathrm{kOe})$. The figure shows a good agreement between calculations and 


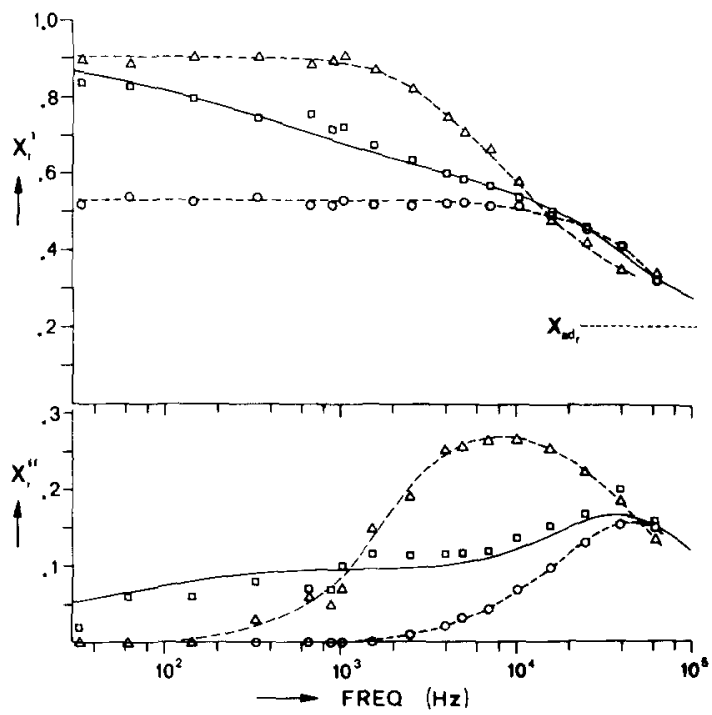

Fig. 1. Dispersion $\chi^{\prime}(\nu)$ and absorption $\chi^{\prime \prime}(\nu)$ curves of $\mathrm{CMN}$ at $4.2 \mathrm{~K}$ and $17.9 \mathrm{kOe}$, relative to $\chi_{\mathrm{T}}(H=0)$. $\square$ : liquid-helium contact, $O$ : vacuum, $\triangle$ : gaseous helium at saturation pressure. The solid line is calculated with the thermal conduction model for liquid helium conditions.

measurements over the entire frequency range and similar results were obtained at lower temperatures. So we may conclude, that the observed relaxation effects are due to the poor thermal conduction of liquid helium.

This conclusion is further supported by the following observation. When the crystal is surrounded by gaseous helium at saturation pressure, the susceptibility remains constant up to $1 \mathrm{kHz}$ and no relaxation effects are encountered at low frequencies (fig. 1). A simple explanation of this phenomenon may be that the thin helium film, that is formed on the crystal surface, has a large effective heat capacity because of evaporation and readsorption of helium and that this adsorbed film is more effective in keeping the lattice temperature constant than bulk liquid helium.

Fig. 1 also shows the results of the vacuum measurements at $4.2 \mathrm{~K}$ and $17.9 \mathrm{kOe}$. From the isothermal and adiabatic limits of the vacuum and saturated pressure dispersion curves the effective lattice specific heat $C_{\mathrm{L}}^{*}$ was calculated to be $1.24 \times$ $10^{3} \mathrm{~J} / \mathrm{m}^{3} \mathrm{~K}$. This value is, within experimental error, equal to the calorimetric value given by Colwell [3]. This result was also obtained at lower temperatures down to $2 \mathrm{~K}$ and therefore we must conclude that in cerium magnesium nitrate all the lattice oscillators participate in the relaxation process.

\section{Conclusions}

1. The poor thermal conduction in liquid helium leads to a bottleneck in the heat transfer between the spin system of CMN and bath. This environmental influence can be explained with a thermal conduction model.

2. A crystal can be effectively cooled by placing it in helium gas at the saturation pressure.

3. Although the Orbach process is the dominant mechanism in the spin-lattice relaxation all lattice oscillators participate in the observed relaxation. No partial lattice participation occurs.

\section{References}

[1] J. Flokstra, G. J. Gerritsma and L. C. van der Marel, Physica 94B (1978) 53.

[2] A. van der Bilt and A. J. van Duyneveldt, Proc. LT 15 (1978) 1002.

[3] J. H. Colwell, J. Low Temp. Phys. 14 (1974) 53.

[4] J. E. Robichaux and A. C. Anderson, Phys. Rev. B2 (1970) 5035. 